American Journal of Engineering Research (AJER)

e-ISSN : 2320-0847 p-ISSN : 2320-0936

Volume-01, Issue-01, pp-01-15

www.ajer.us

\title{
BROADBAND CABLE ACCESS NETWORKS FOR TRIPLE PLAY SERVICES: SOURCE-DESTENATION
}

\author{
Nasser N. Khamiss \\ Information Engineering Collage, Al-Nahrain University, Iraq
}

\begin{abstract}
This paper evaluates the performance of sending Triple play Services over hybrid networks. The network performance factors will be considered by observing the network's availability, packet loss, delay and throughput. These evaluations will be tested over different network scenario, where the last mile media services suggested to be delivered over telephone twisted pair by means of using ADSL. The attention will be concerned for services over cable, where a proposal of using bandwidth efficient turbo trellis coded modulation (TTCM) in ADSL DMT systems instead of the multidimensional 16-state Trellis Coded Modulation (MTCM) that is given as an option in Asymmetric Digital Subscriber Lines (ADSL) standard based on discrete multitone (DMT) techniques. The results show that by using turbo codes, it can obtain $6 \mathrm{~dB}$ coding gain for a bit error rate (BER) of $10^{-6}$ in AWGN channels and more than $6.8 \mathrm{~dB}$ coding gain for a BER of $10^{-7}$ using a concatenated coding scheme.
\end{abstract}

Keywords: Triple Play, ADSL2+, IPTV, VoIP, QoS, and Network Availability

\section{INTRODUCTION}

Generally triple play architecture consists of the Service Provider Network, the core network, the access network where end users reside and the equipments at the subscriber's home. A Triple Play solution can distribute 50 to $150 \mathrm{TV}$ channels over an IP network with voice over IP and high-speed Internet. Services of video, voice, and data can be sent from the IP head-end using an IP core network over an optical backbone network to the central office (CO) [Michael 10]. The CO relies the data to the access network (AN) in which digital subscriber line access multiplexers (DSLAMs) will be proposed to home`s services requirements. From the technoeconomic evaluation of telecommunications market studies, there are an addressing of a wide range usage of telecommunication networks due to major business cases [Borgar 06]. Asymmetrical Digital Subscriber Lines (ADSL), as an access technology over the existing nonloaded copper loop plant, are intended to provide up to $8 \mathrm{Mbps}$ downstream digital transport from central office to customers and up to 640kbps upstream transmission [Nasser 09]. Such an asymmetric transmission has potential usage in services like advanced videotext, compressed TV quality video and distant education applications, where most of the information goes from the service providers to the customers. Forward error correcting codes (FEC) are employed in communication systems achieve coding gain to increase the system margin and the maximum achievable transmission rate [Neubauer 06]. A four dimensional version of MTCM concatenated with RS code was proposed previously for ADSL DMT systems to provide about $5 d B$ coding gain without bandwidth expansion [Moreira 06]. Further improvement is very difficult to obtain because of the complexity of the Viterbi decoding (VA) for the MTCM. A new powerful coding scheme, turbo coding, has the potential of providing near Shannon limit performance with reasonable complexity in AWGN channels. Therefore, applying turbo codes in ADSL DMT systems is now a challenging practical problem.

\section{TRIPLE PLAY NETWORK TOPOLOGY}

The major components are the backbone and aggregation networks, which consist mainly of server head end, external Internet peers, IP core, Broadband Remote Access Server (BRAS) edge, and ethernet 
aggregation network, like DSLAMs, local loop, and the customer household [Michael 10], see Figure (1). The next sections will define the important components that are related to this work.

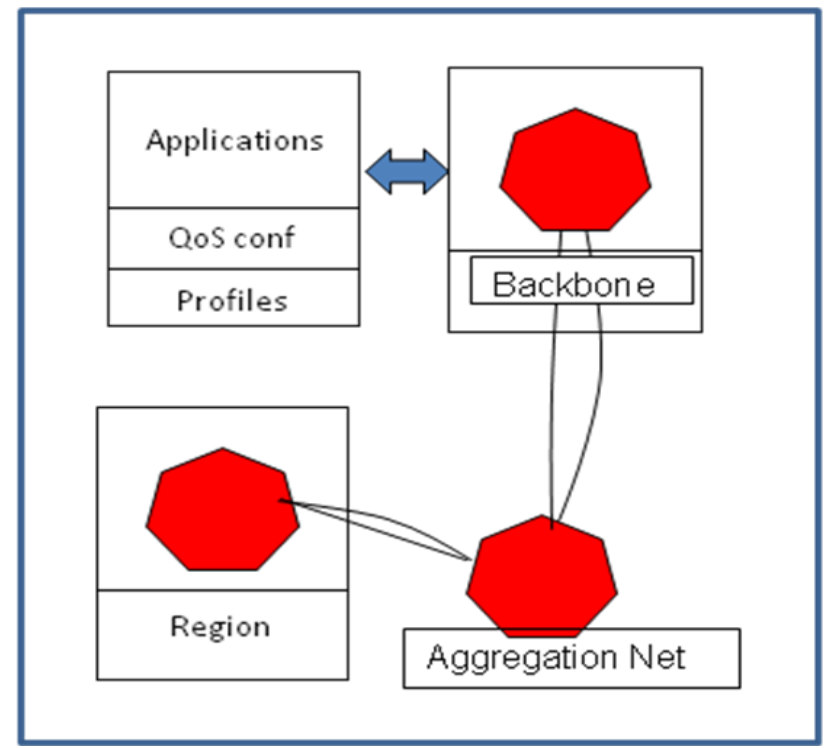

Figure 1 Network topology

\subsection{Backbone Subnet:}

In Figure (2) the "Backbone" subnet is designed with 4 servers configured to stream stored audio and video contents, HTTP and FTP. It contains a 100Mbps IP network and access routers for both IP Multicast traffic load (R3, RP, and R4) and IP Unicast traffic load (DSR) these routers are connected to the switches (Source and PPP) which are divided the traffic into VLANs. Then these routers are connected to the BRAS router at Aggregation subnet through a PPP_SONET_OC24 with data rate (1244.16 Mbps) wide area network (WAN) link. The approximate distance between the backbone subnet and Aggregation subnet is $5.44 \mathrm{~km}$, which corresponds to approximately $1.813 \mathrm{~ms}$ propagation delay. The multicast and unicast techniques are configured with VLAN parameter to have been efficiency triple play services. The network topology of this work will be explained in the next paragraphs.

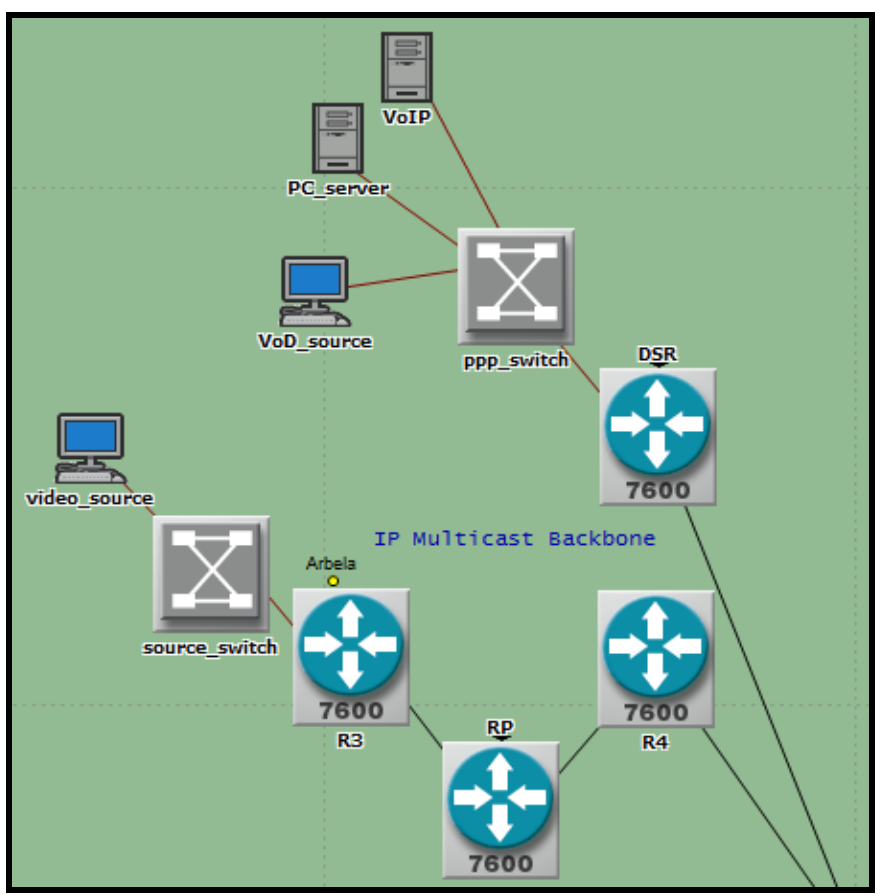

Figure 2 Backbone Subnet 
Multicast technology protocols must be presented in the first IP network at the core, edge, and access layers of an IP ADSL triple-play network for supporting IPTV services. Where multicast is enabled in routers by configuring it using the attribute "IP multicast -> IP multicast parameter -> multicast routing"

\subsection{Aggregation network Subnet:}

The "Aggregation" subnet, that receives all traffic from backbone network by using PPP_SONET_OC24 with data rate (1244.16 Mpbs), delivery it to access network at a customer side by using Metro Ethernet Network [Gallant 06]. This subnet has several components as show in Figure (3); these components are discussed next subsections.

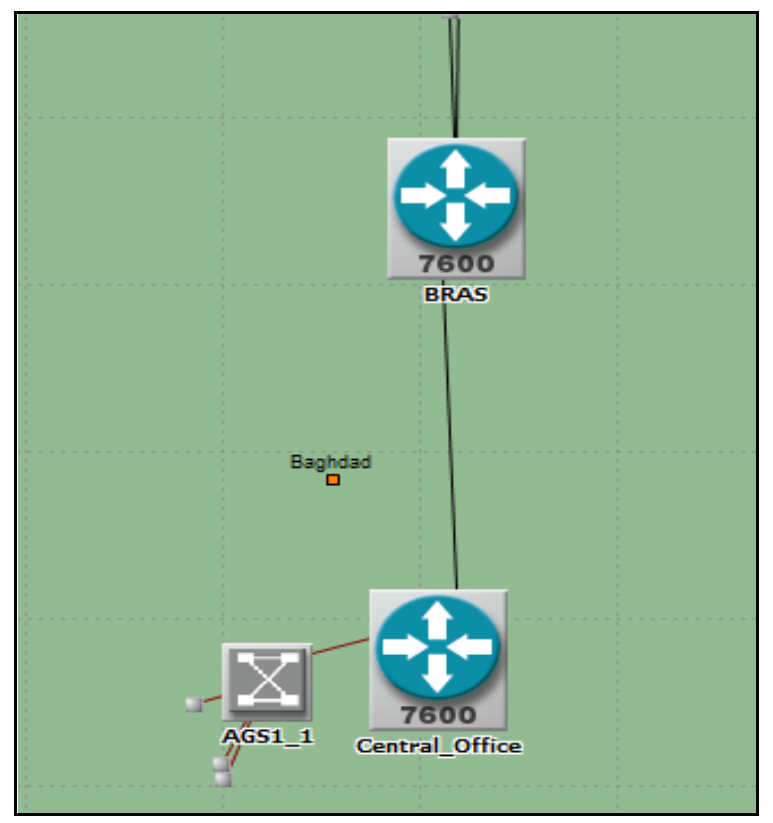

Figure 3 Aggregation_network Subnet

\subsubsection{BRAS}

The "BRAS" is a Broadband Remote Access Server router that forwards packets between the core and customer. It is a complex router that implements dynamic per-subscriber IP policies, Quality of Service (QoS) profiles, rate limiters, packet manipulation, address assignment, session termination and forwarding.

\subsubsection{Center Offices (CO)}

The $\mathrm{CO}$ router relies the data to the access network (AN) which consists of digital subscriber line access multiplexers (DSLAMs) and broadband digital loop carriers (DLCs). There it has the so-called last mile distribution of the service (i.e. video, voice or data) which afterwards enters the subscriber's home through the modem. Central Office router and Remote DSLAMs at the region subnet are supplied with Gigabit Ethernet links.

\subsubsection{Metro Ethernet Network:}

DSLAM are aggregated by Aggregation Switches also called (metro Ethernet network). A metro Ethernet network is useful when a switched layer between the DSLAM at Region subnet and the CO router in Aggregation subnet provides cost-effective aggregation capacity. Such a scenario would arise if the bandwidth utilization per-DSL-port is not enough to justify connecting DSLAMs directly to the CO. This benefit needs to be weighed against the expected traffic loads to and from DSLAMs. Also consider whether a switch can continue to offer enough statistical traffic multiplexing gains in the future. Metro Ethernet is replacing SONET links to Gigabit Ethernet links (1000Basex_adv) to flexibly and cost effectively scale the network to support increased video traffic. Another drawback to SONET in the access network is that the available feeder bandwidth has only recently upgraded to OC-3 (155 Mbps). These networks require other costly upgrades to get to OC-12 (622 Mbps), and even newly planned networks at OC-48 (2.4 Gbps) will be limited if VoD and higher speed data services achieve their expected growth. The available bandwidth divided among all of the triple-play services and video services alone so that Ethernet link 1000Basex_adv with data rate $1 \mathrm{Gpbs}$ in this network is used. 


\subsubsection{Access Network (Region Subnet)}

This network shows an example for the delivery of Triple-Play services (Data, Voice and Video) over Asymmetric Digital Subscriber Line (ADSL) with data rate (downstream 12Mpbs/ upstream 1.3Mpbs). It simulates end-to-end communications between residential customers and backbone network.

\section{ADSL DMT TRANSMISSION MODEL}

Figure (4) shows a typical configuration of ADSL DMT system. The proposed FEC structures of the transmitter and receiver of ADSL DMT system is shown in Figure (5) and figure (6) respectively. The input bits are first RS coded. With the bit allocation table, the transmitter then allocates the corresponding number of bits to the subchannels. The turbo encoding/decoding operates across all the subchannels, followed by the constellation mapping. TTCM is thus performed by proper combination of the turbo coding and the constellation mapping.

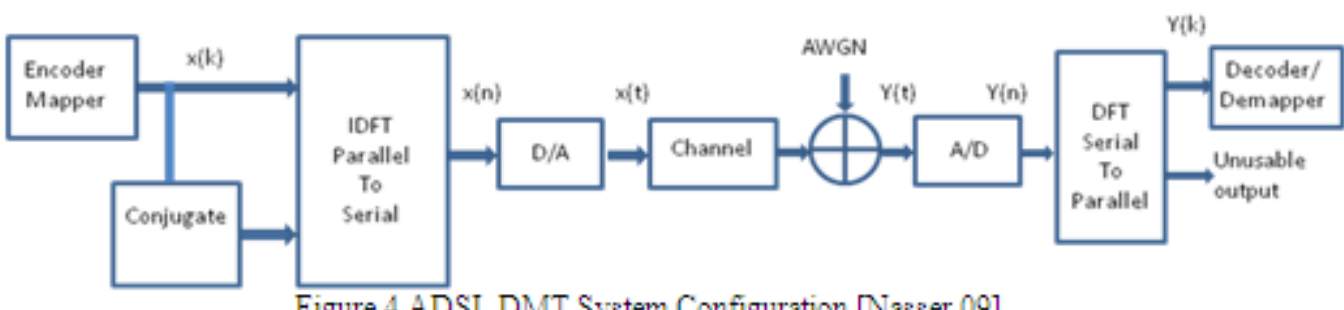

Figure 4 ADSL DMT System Configuration [Nasser 09]

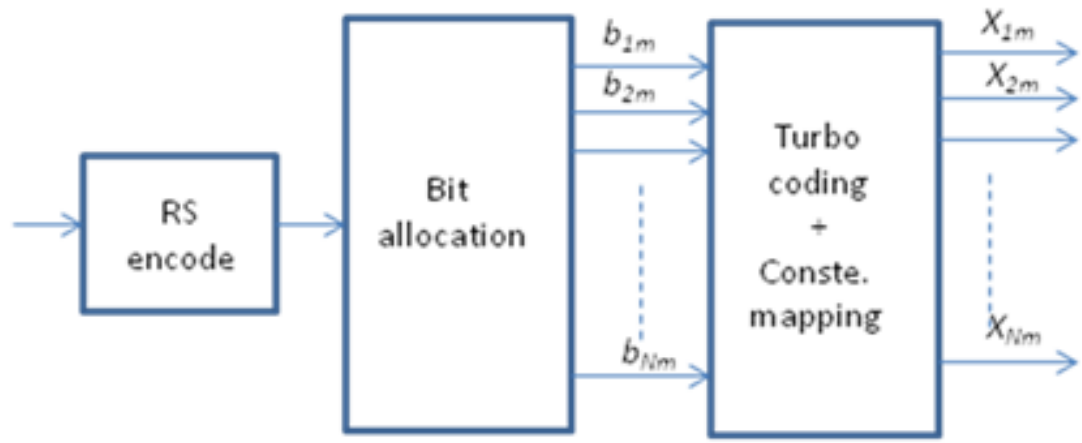

Figure 5 System encoder

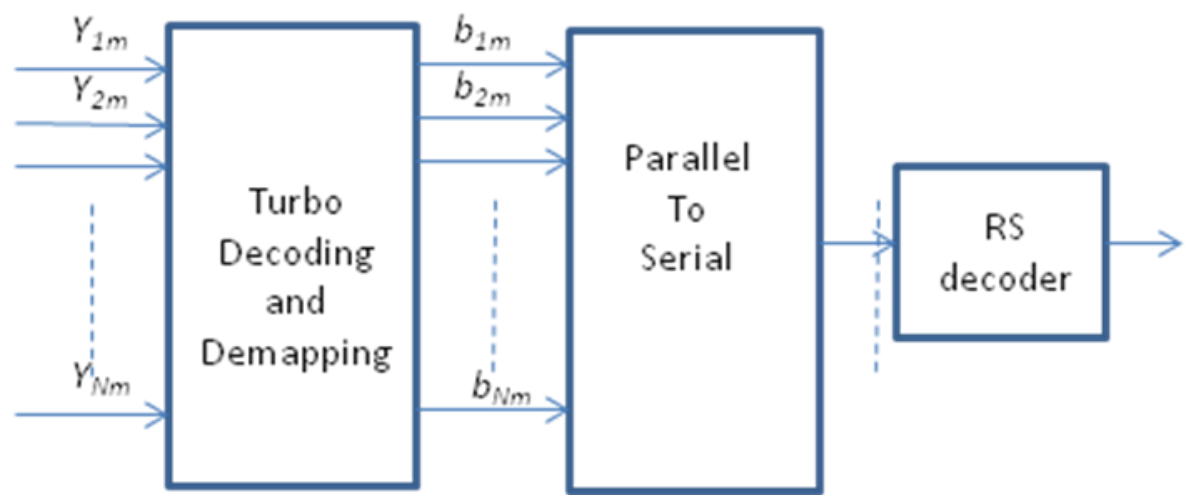

Figure 6 System decoder

\subsection{Encoding Operation layer design:}

In this section a method of providing forward error correction for data services uses a parallel concatenated convolutional code which is Turbo Code is used. An encoder of a parallel concatenation (PCCC) of typically two systematic is used [Moreira 06]. Which consists of recursive convolutional codes ("constituent codes") separated by an interleaver that randomizes the order of presentation of information bits to the second constituent encoder with respect to the first constituent encoder, see Figure (7). The two encoders are identical and built based on the RSC encoder of Figure (8). The performance of a Turbo Code depends on the choice of constituent codes, interleaver, information block size (which generally increase with higher data rates), and 
number of decoder iterations. For a particular Turbo Code, in which the constituent codes are fixed, one can ideally adjust the block size and number of decoder iterations to trade-off performance, latency, and implementation complexity requirements. As the block size changes, however, a new interleaver matched to that block size is required.

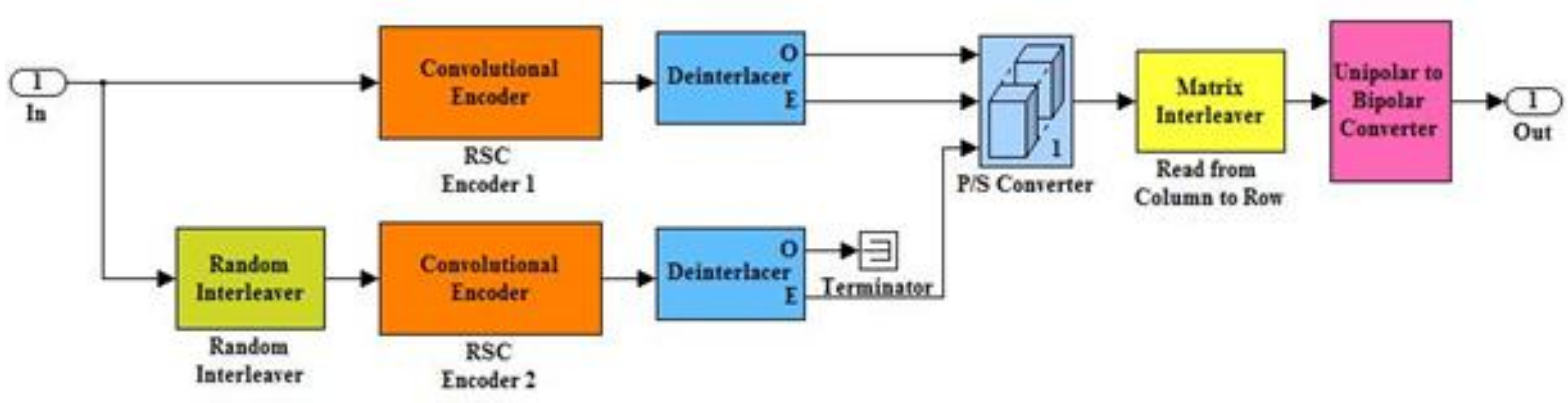

Figure 7 Turbo code encoder

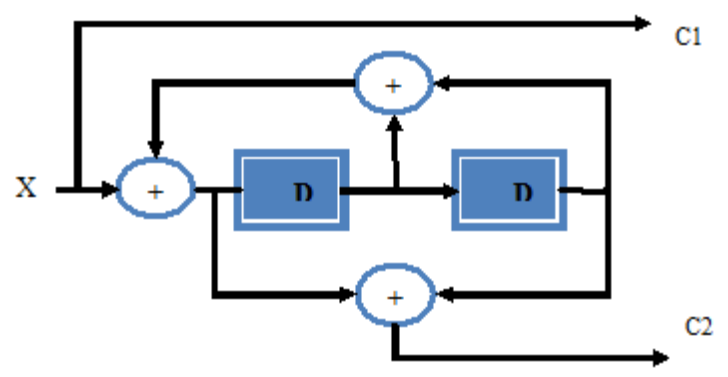

Figure 8 The RSC encoder with $r=1 / 2$ and $\mathrm{K}=3$

In this work the code rate of $1 / 2$ and $1 / 3$ is considered to get the proper decision making of bandwidth efficiency and system performance. The information bits are always transmitted across the channel. Depending on the desired code rate, different code rates are achieved by puncturing the parity bit sequences from the two constituent encoders. As the code rate increases, the bandwidth efficiency will be improved and the performance is degraded since the decoder has less information to use in making a decision. Therefore, a tradeoff must be made between the code rate and the performance.

The deinterlacer block accepts the input vector that has an even number of elements and alternately places the elements in each of two output vectors. Therefore it is used to separate the systematic and the parity bit of each RSC encoder. As mentioned previously, the systematic bit of the second encoder is nothing more than a repetition code, thus a termination block is used on the odd output of the second encoder. The three streams: the systematic bits and the two parity bits are concatenated using vertical concatenation. A matrix interleaver is used to perform block interleaving by filling a matrix with the input symbols row by row and then sending the matrix contents to the output port column by column so as to avoid burst errors. The output then forwarded to the puncture block which will periodically remove bits from the encoded bit stream, thereby increasing the code rate.

\subsection{Turbo Decoding}

The truly unique aspect of Turbo codes is their iterative decoding process. The iterative decoding structure consists of two Soft-Input, Soft-Output (SISO) decoding modules that are separated by a pseudorandom interleaver/deinterleaver. The performance analysis of Turbo codes always assumes the usage of a Maximum Likelihood (ML) decoder at the receiver for efficient data recovery. The output of each encoder depends on the last input bit and the generator matrix, which enables the encoding process of the Turbo code to be represented by two joint Markov processes. It is possible to decode Turbo codes, first by independently estimating each process and then refining the estimates by iteratively sharing information between two decoders [Avril 07]. Since the two processes run on the same input data, it means that the output of one decoder can be used as a priori information by the other decoder.

It is necessary for each decoder to produce soft-bit decisions in order to take advantage of this iterative decoding scheme. The soft-bit decisions are usually in the form of Log Likelihood Ratios (LLRs). The LLR data 
serves as the a priori information and is defined as the likelihood of the received bit being a one rather than a zero where the decision 1 is made for a positive LLR and the decision 0 is made for the negative LLR.

A decoder that accepts input in the form of a priori information and produces output in the form of posteriori information is called a Soft Input Soft Output (SISO) decoder. The inputs to the decoder are systematic data, parity data and the a priori data from the previous decoder and the output of the decoder is the LLR data. The generic block diagram of a SISO decoder is shown in Figure (9).

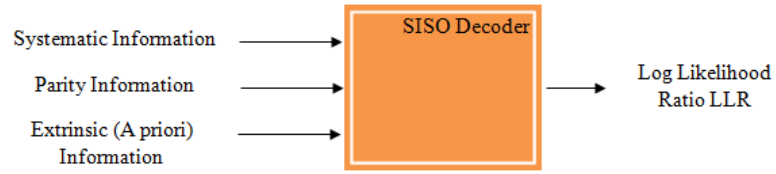

Figure 9 Block digram of SISO decoder

\subsubsection{Operation of Turbo Decoding}

A block diagram of a Turbo decoder is shown in Figure (10) which consists of two component decoders - decoder \#1 to decode data sequences from encoder 1 and decoder \#2 to decode sequences from encoder 2.

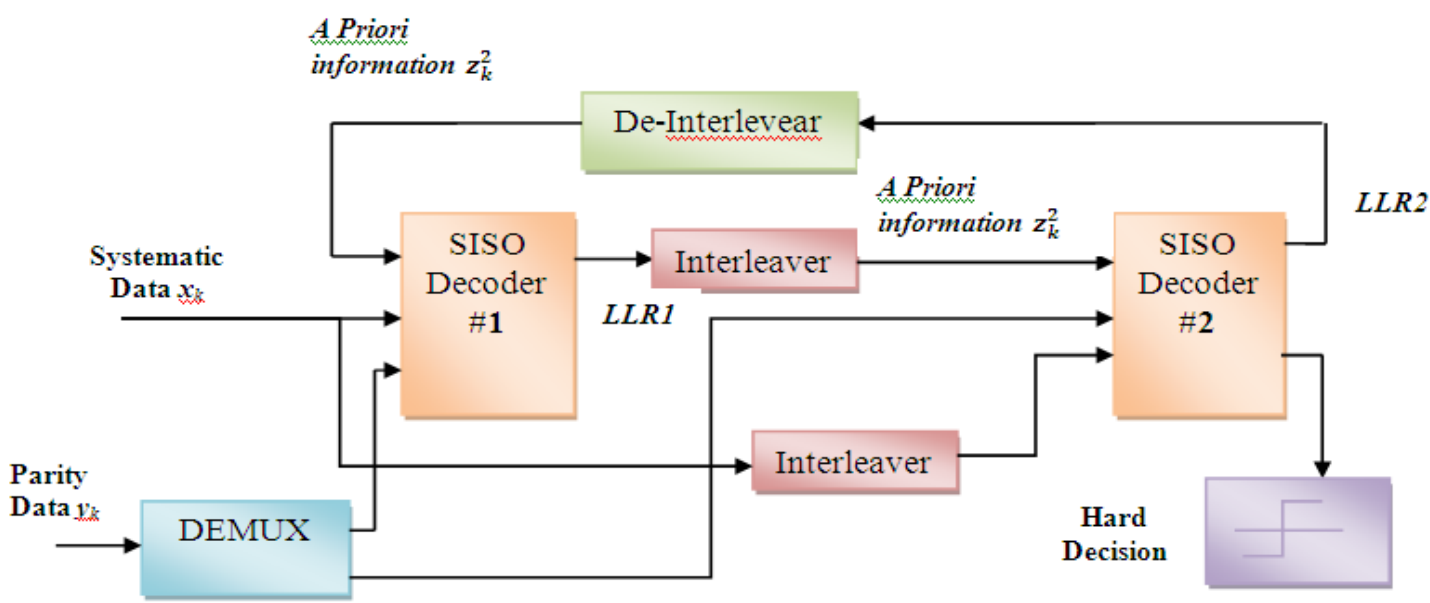

Figure 10 Turbo decoder

The first decoder operates on the systematic channel observation $y_{k}^{0}$, the parity channel observation from the first RSC encoder $y_{k}^{1}$ and the a priori information $z_{k}^{1}$. The a priori information for SISO (decoder \#1) is initially set to all zeros, since the second decoder has not produced any information. This implies that each information bit is equally likely to be a 0 or a 1 initially. Both channel observations are multiplied by the channel reliability $L c=4 a E s / N o$ where the variable $a$ is the fading amplitude, $E s$ is the average symbol energy and $N o$ is the noise Power Spectral Density (PSD). The channel reliability places more emphasis on the channel observation when the SNR is high and there is no fading. Likewise, more emphasis is placed on the a priori information $Z_{k}$ when the SNR is poor or when there is a deep fade.

The output of the SISO decoders is expressed as a Log-Likelihood Ratio (LLR) $\Lambda_{k}$ where the decoder's output at time $k$ can be broken down into three distinct parts: the scaled systematic channel estimate $\frac{4 a E_{S}}{N_{0}} y_{k}^{0}$, the $a$ priori information $Z_{k}$ and the extrinsic information $l_{k}$.The $k^{t h}$ LLR is expressed as:

$\Lambda_{k}=\frac{4 a E_{s}}{N_{o}} y_{k}^{o}+z_{k}+l_{k}$

The extrinsic information is the new information generated by the current stage of decoding. In Turbo decoder, the extrinsic information for the first decoder is determined by subtracting the systematic channel observation and the current stage's a priori information from the LLR $\Lambda_{k}^{1}$, thereby preventing positive 
feedback. The extrinsic information is then permuted by pseudo-random interleaver and used as the weighted $a$ priori information for the second decoder. The second decoding module operates on the weighted a priori information from first decoder $z_{k}^{2}$, the permuted channel observation $y_{k}^{0}$ and the parity channel observation from the second RSC encoder $y_{k}^{1}$, that generate a new LLR $\Lambda_{k}^{2}$.

The first decoder is presented with the result from the second decoder one can imagine that it might improve its performance, compared to its first decoding attempt. The two decoders iteratively exchange this extrinsic information and improve their estimates about the decoded bits. If all the decoding iterations have been completed, the final output $\Lambda_{k}^{2}$ is deinterleaved and hard-limited to produce the final decision. The iterative decoding process improves the BER performance of Turbo codes in a superior way [Neubauer 07]. As stated before, when Berrou and Glavieux achieved BER $=10^{-5}$ at $E s / N o$ within $0.7 \mathrm{~dB}$ of the Shannon limit using a rate $1 / 2$ turbo code, they used 18 decoding iterations.

\subsubsection{Parallel Concatenated Code}

The receiver error handling mainly consists of two parts, which are the Receiver Front End and the Turbo Decoder block as shown in Figure(11).

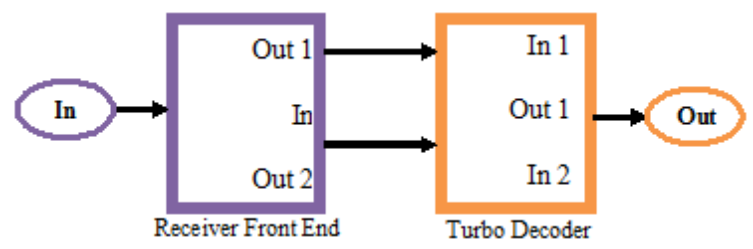

Figure 11 Turbo Decoder Main Blocks

As shown in Figure (12), the data is divided by the noise variance through the gain block, then sampled and held for a specified sample period by the zero-order hold block. After that, a matrix deinterleaver block is used which fills the input symbols into a matrix column by column and then sending the matrix contents to the output port row by row.

Two interlacer blocks are used to reconstruct the data as produced by the two de-interlacer in the transmitter side, then the output of the both interlacer is forwarded to the Turbo decoder block. Figure (13) shows the PCCC decoding process that consists of two APP decoder blocks, a random interleaver and a feedback loop. As in SCCC, these blocks form a loop and operate at a rate of six times faster than the encoding portion.

The error rate block is the same as that used in SCCC system. As shown in Figure (14), the data are sampled and held for the specified sample period by a zero-order hold block. The error rate is calculated for all iterations by comparing the received data with the transmitted data and the output is converted to six independent channel samples. Then a mean block is used to return the mean of the input elements over the time. Finally the display block shows error rates of the six iterations where the final BER is obtained from the last iteration.

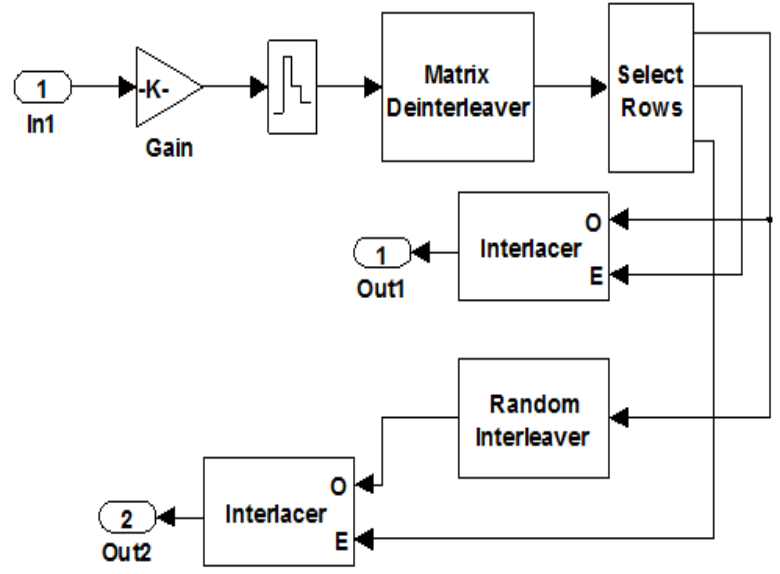

Figure 12 Receiver front end 


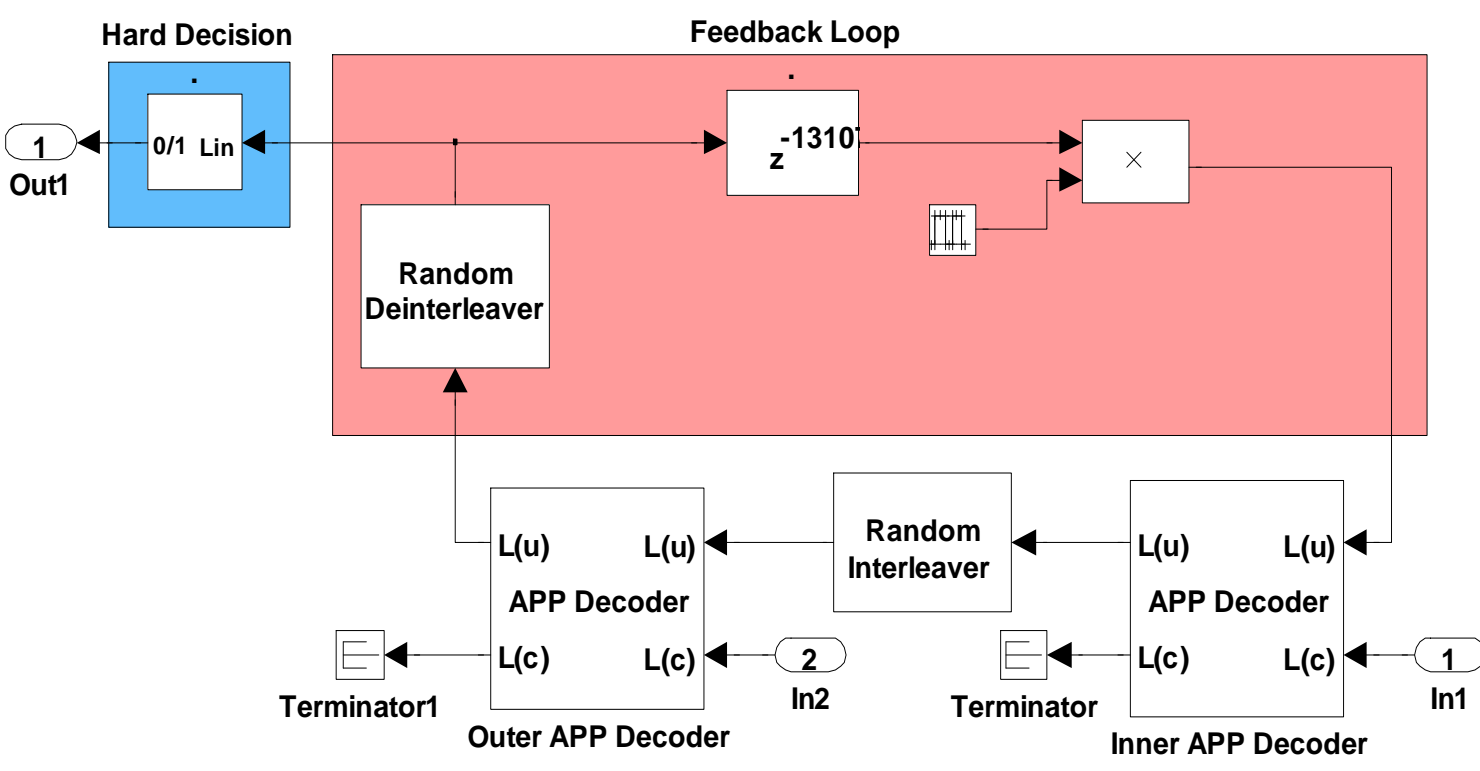

Figure 13 Turbo code decoder

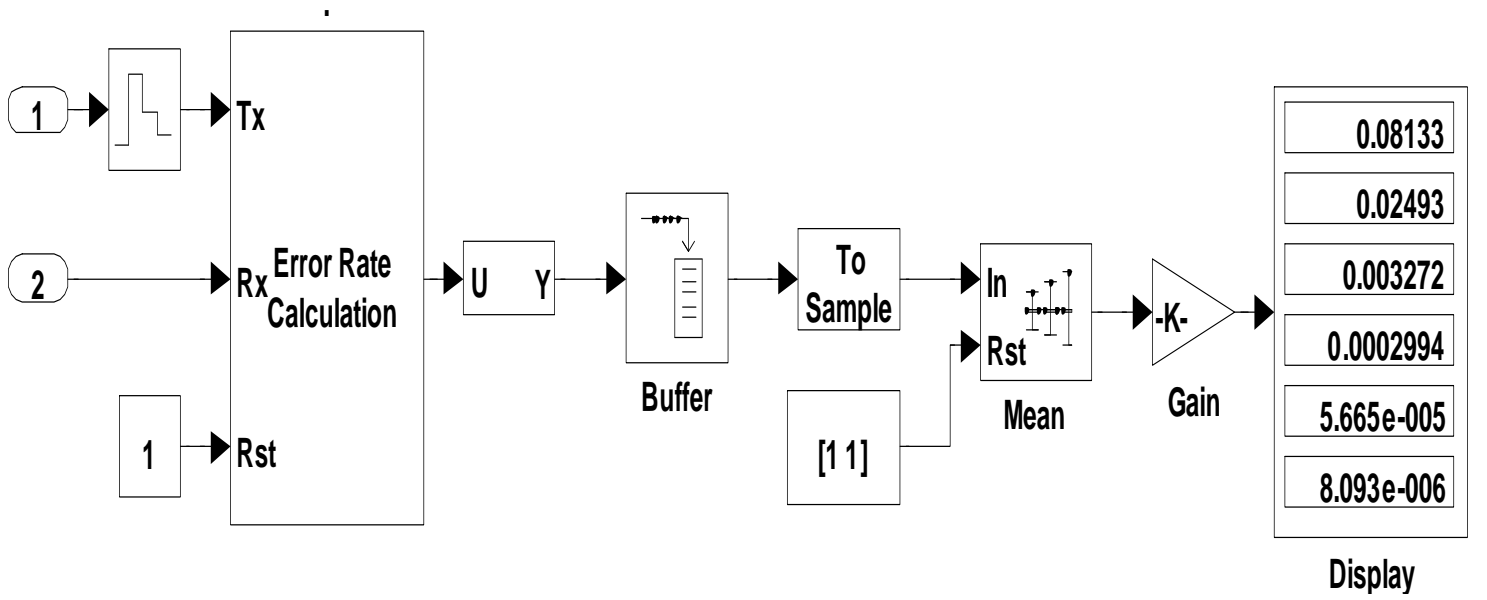

Figure 14 Multiple iterations error rate calculation

\section{SYSTEM TEST AND RESULTS}

The system test will be expressed as ADSL DMT part to show the last mile network quality improvement, while the other part of the system test will give the overall system QoS that includes the evaluation of system QoS factors.

\subsection{Simulation Results of using TTCM in QAM Transmission in ADSL DMT Systems}

These simulations of modem apply DSL based on DMT system and turbo code. In this system four different services will be supported for a user by using modem with different code rate. The results of data bits performance are shown in Figure (15) and Figure (16) by using code rate 1/2 and 1/3 respectively. System with rate $\mathrm{R}=1 / 2$ can be achieved by puncturing the parity bits of the RSC encoders. The performance of each service depends on the channel characteristics in the presence of the turbo decoder on correcting the errors. A code gain of $6 \mathrm{~dB}$ for system using code rate 1/3 is achieved as shown in Figure (17). This figure also shows the code gain will decrease as the number of iteration is increase. The Figures (18) and (19) show the effect of turbo coding on the data bits, where the BER performance of the system will increase as the iteration increases. As noticed the increasing in coding rate achieves better performance than that of using lowest coding rate, when using code rate $1 / 2$ data rate reaches to $8.8 \mathrm{Mbps}$ ( 2.4 for each service), while when code rate $1 / 3$ is used the transmitted data rate reached to $14.8 \mathrm{Mbps}$ (3.7 for each service) in this system. 


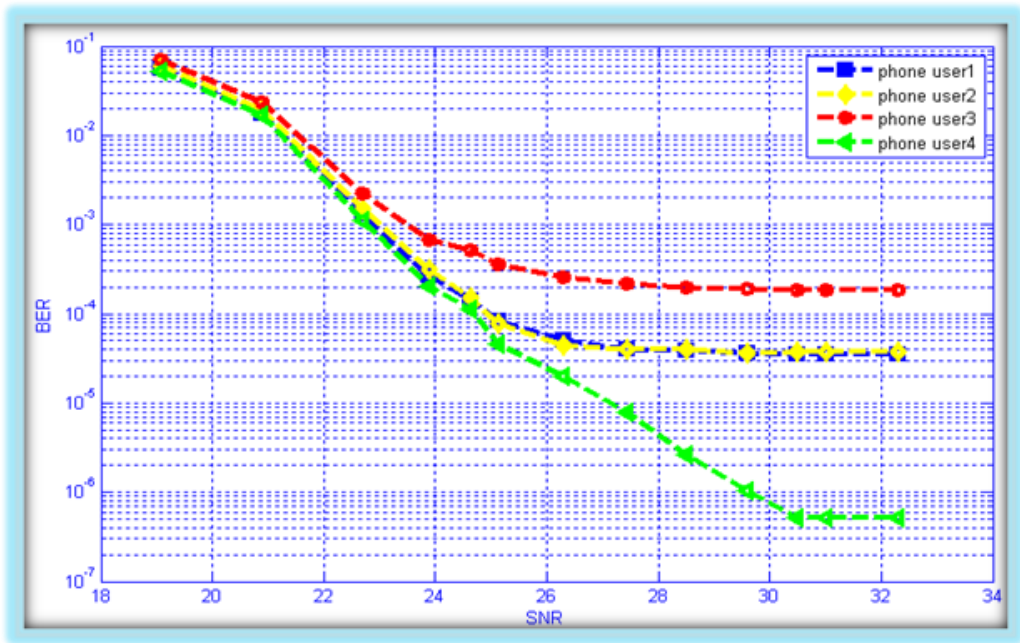

Figure (15): BER of the four service line users using turbo code rate $1 / 2$

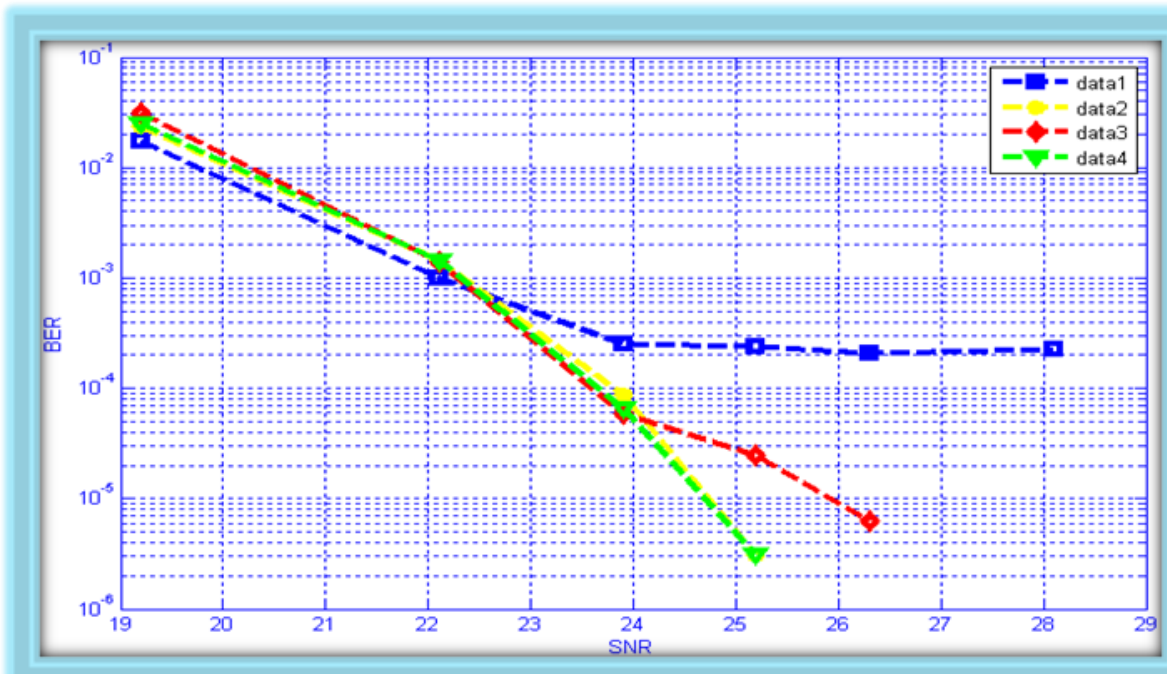

Figure (16): BER of the four service line users with code rate 1/3

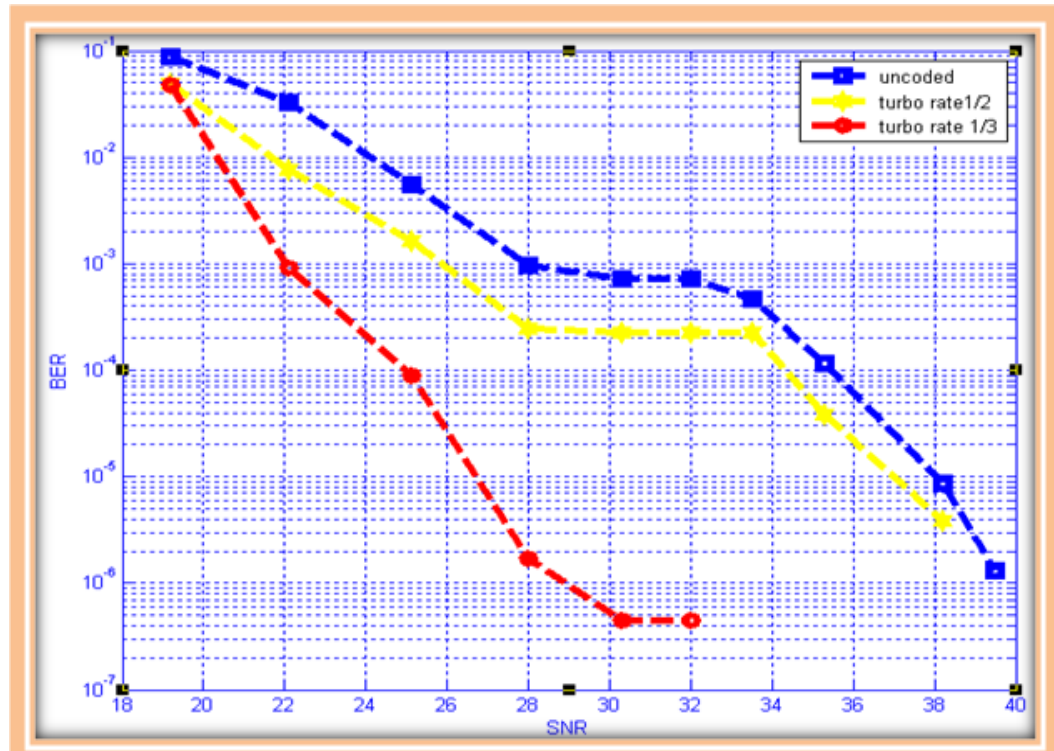

Fig (17) BER comparison of un-coded with different Turbo code rate (1/2 and 1/3) 


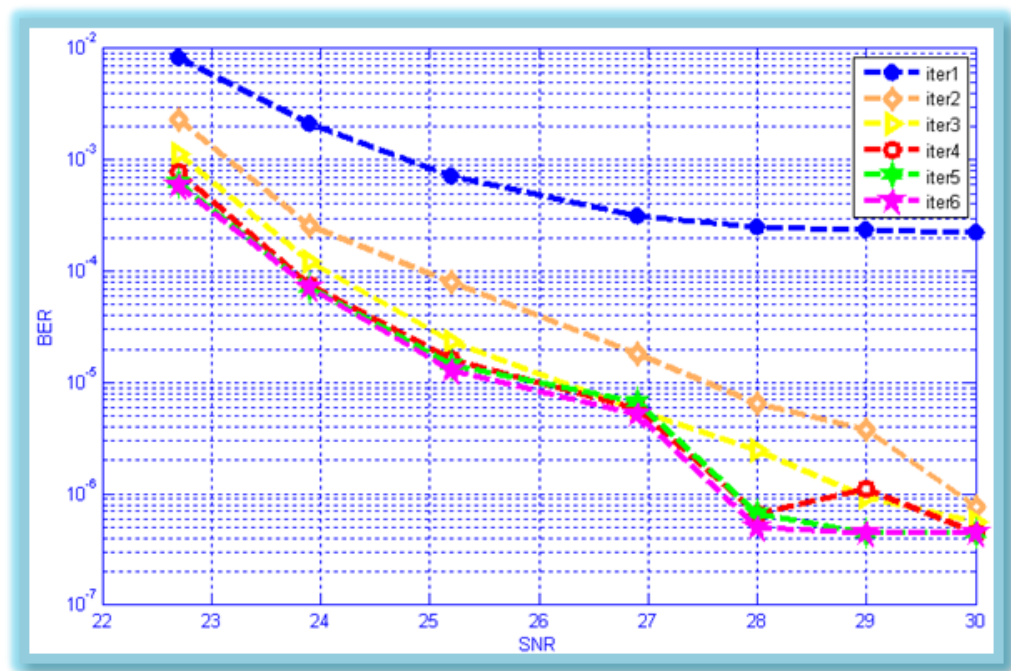

Fig (18) BER of turbo decoding process (code rate 1/2) on both data bits and phone users bits

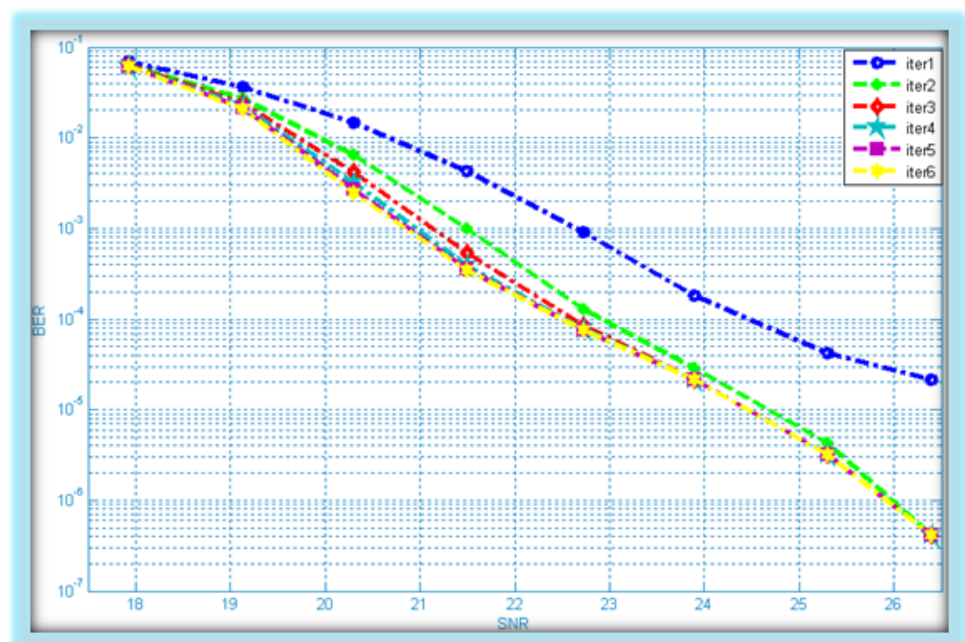

Fig(19) BER of Turbo decoding process (code rate 1/3) on both data bits and phone users bits

\subsection{Network Measurements}

In this section the performance measurements factors [Kurose 10] of End to End Delay as given in $\mathrm{Eq}(1)$, Jitter as given in $\mathrm{Eq}(2)$, Packet Loss Ratio as given in $\mathrm{Eq}(3)$, and the Throughput as given in Eq(4) will be tested over an integrated network from the delivery source of triple play services to the destination user where the DSL is the mean media of last mile. At this test a service of VoD, IPTV, VoIP, HTTP, and FTP from source to destination will be demonstrated and measured corresponding to the above factors.

$d_{\text {endend }}=Q\left(d_{\text {proc }}+d_{\text {queue }}+d_{\text {trans+ }} d_{\text {prop }}\right)$

$Q \quad$ network number elements

$\boldsymbol{d}_{\text {proc }} \quad$ processing delay at network element

$\boldsymbol{d}_{\text {queue }} \quad$ queuing delay at network element

$\boldsymbol{d}_{\text {trans }} \quad$ transmission time of a packet over link

$\boldsymbol{d}_{\text {prop }} \quad$ propagation delay across network link

Jitter $=(t 4-t 3)-(t 2-t 1)$
$t 4-t 3$
is the expected packet reception time
$t 2-t 1$
is the actual packet reception time

Negative jitter means that the packets where received in different time range i.e $(\mathrm{t} 4-\mathrm{t} 3)<(\mathrm{t} 2-\mathrm{t} 1)$.

\section{$P L R=\frac{\text { lost_packets }}{\text { lost_packets }+ \text { received packets }}$}

Another variation of this metric is the media loss rate (MLR) which track packet loss over time: 


\section{$P L R=\underline{\text { excepted_packets }- \text { received_packets }}$ time_unit $\mathbf{R}_{\min }=\frac{(\text { packets_size_in_bytes })(\text { number_of_packets_in_second })(8 \text { bit } / \text { bytes })}{\text { second }}$}

The QoS recommendations adopted by IPTV and voices service providers, Y.1541, will be considered in the term of system test. In additional to that the FTP downloads response time, HTTP page, Buffer Overflow Percentage response time, Free-space Pathloss, and the Erceg path loss model will be also observed. An Application Parameter of the type of services of voice Application, HTTP Application, FTP Application (File sharing /downloading), IPTV application, and VoD application are given in tables (1-5) respectively [PATRICK 07].

Table1: Residential VoIP application characteristics

\begin{tabular}{|l|l|l|l|l|}
\hline $\begin{array}{l}\text { Encoder } \\
\text { Scheme }\end{array}$ & $\begin{array}{l}\text { Voice Frame } \\
\text { Per Packet }\end{array}$ & $\begin{array}{l}\text { DSCP } \\
\text { Value }\end{array}$ & Compression Delay(s) & $\begin{array}{l}\text { Decompression } \\
\text { Delay(s) }\end{array}$ \\
\hline G.711 & 1 & EF & 0.02 & 0.02 \\
\hline
\end{tabular}

Table2: Residential HTTP application characteristics

\begin{tabular}{|l|l|l|}
\hline Http Specification & Object Size(Byte) & DSCP Value \\
\hline Http 1.1 & $\begin{array}{l}\text { Constant(1) } \\
\text { Media Image (0.5-2) } \\
\text { Large Image (2-10) }\end{array}$ & \\
\hline
\end{tabular}

Table3: Residential FTP application characteristics

\begin{tabular}{|l|l|l|}
\hline Download & Size File (MB) & DSCP Value \\
\hline $100 \%$ & 5 & AF13 \\
\hline
\end{tabular}

Table4: IPTV application characteristics

\begin{tabular}{|l|l|l|l|}
\hline Frame Rate & $\begin{array}{l}\text { Incoming Frame } \\
\text { Size (Byte) }\end{array}$ & $\begin{array}{l}\text { Outgoing Frame Size } \\
\text { (Byte) }\end{array}$ & DSCP Value \\
\hline $15 \mathrm{Fps}$ & 17280 & 17280 & AF33 \\
\hline
\end{tabular}

Table 5: VoD application characteristics

\begin{tabular}{|l|l|l|}
\hline Parameters & T2 & Matrix III \\
\hline Resolution & $\mathbf{1 2 8 0 x 7 2 0}$ & $\mathbf{3 5 2 x 2 8 8}$ \\
\hline Codec & MPEG-2 & MPEG-4 Part 2 \\
\hline Frame Compression Ratio & 58.001 & 47.682 \\
\hline Minimum Frame Size (bytes) & 627 & 8 \\
\hline Maximum Frame Size (bytes) & 127036 & 36450 \\
\hline Mean Frame Size (Bytes) & 23833.792 & 3189.068 \\
\hline Display Pattern & IBBPBBPBBPBB & IBBPBBPBBPBB \\
\hline Transmission Pattern & IPBBPBBPBBIB & IPBBPBBPBBIB \\
\hline Group of Picture Size & 12 & 12 \\
\hline Frame Rate (frames/sec) & $\mathbf{3 0}$ & $\mathbf{2 5}$ \\
\hline Number of Frames & 324,000 & 180,000 \\
\hline Peak Rate (Mbps) & 30.488 & 7.290 \\
\hline Mean Rate (Mbps) & $\mathbf{5 . 7 2 0}$ & $\mathbf{0 . 6 3 7}$ \\
\hline DCSP & $\mathbf{A F 3 3}$ & $\mathbf{A F 3 3}$ \\
\hline
\end{tabular}

For an IP network simulator based to OPNET Modeler 14.5, a Profile Parameters (ftp profile, http profile, voip profile, iptv_ch1 profile, iptv_ch2 profile, iptv_ch3 profile, iptv_ch4 profile, iptv_ch5 profile, iptv_ch6 profile, vod_1 profile, vod_2 profile, vod_3 profile) as given in Figure (20) will be created. 


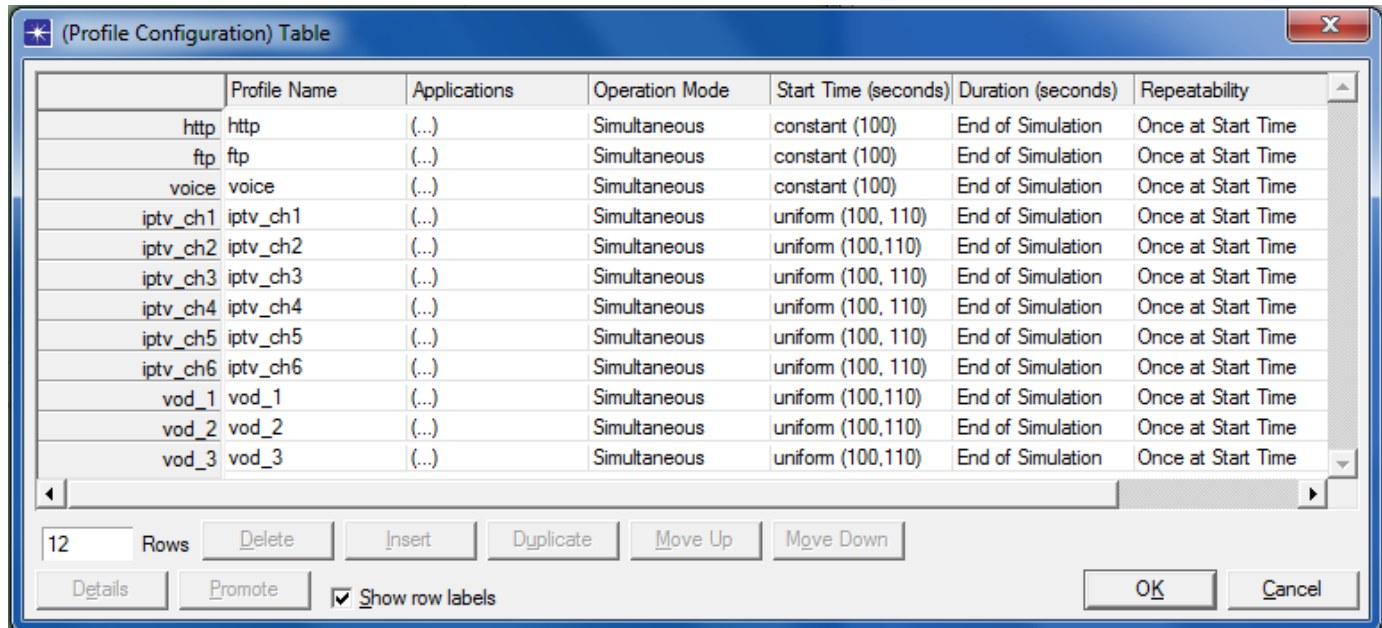

Figure (20) The profile attribute configuration

The QoS Attribute Configuration object defines the CAR, FIFO, WFQ, Custom Queuing, and Priority Queuing is given in Figure (21).

In order to configure the QoS queue priorities in the IP DiffServ the following three schemes are also considered for the purposes of evaluation and compression:

1. Priority Queuing (PQ): which guarantees absolute priority for the voice traffic flow.

2. Weight Fair Queue (WFQ) scheme: which assigns a portion of the total bandwidth to each traffic flow, according to its weight.

3. Low Latency Weight Fair Queuing (WFQ-LLQ): is a complex scheme, where the voice traffic flow has an absolute priority over the other two traffic flows (like in Q), but the video and data traffic flows are served by a WFQ algorithm.

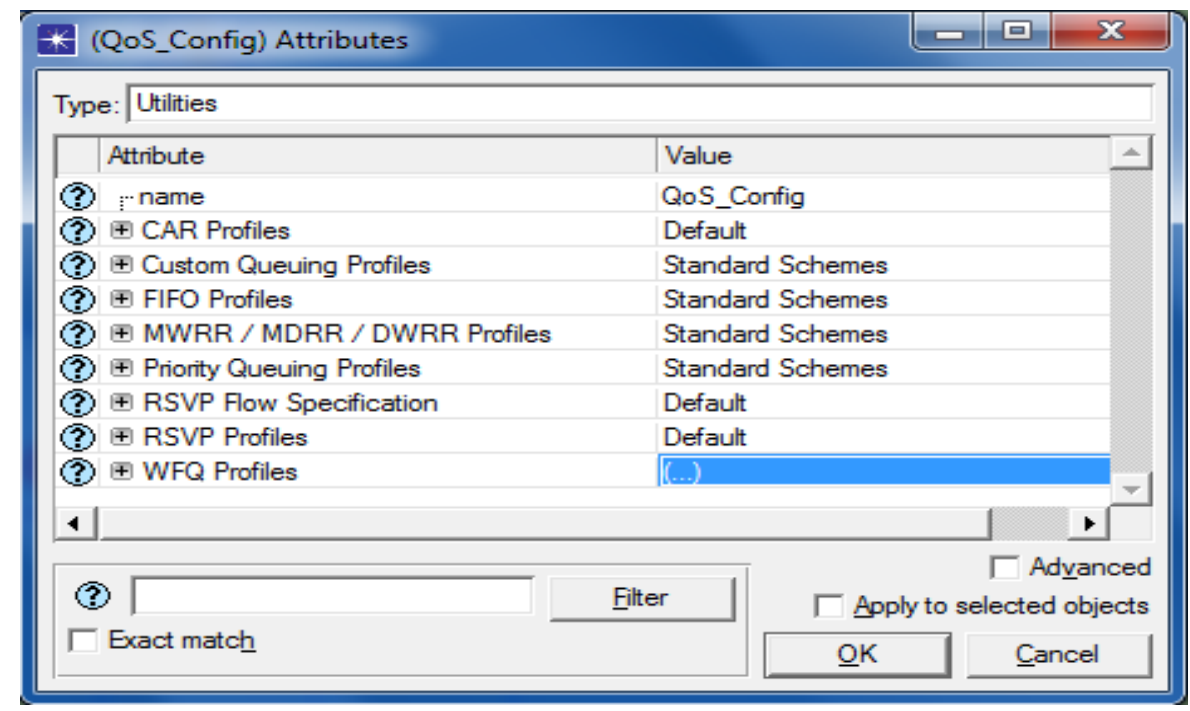

Figure (21) The QoS technologies

\subsubsection{Required Network Simulation and test}

The network simulation and test will be achieved corresponding to the above mentioned network applications parameters. A customer household of 30 houses all received full triple play services simultaneously, where video streams MPEG-2 and MPEG-4 at the source are available will be used to test the network QoS.

To extend the test of the network the backbone subnet of figure (2) is used to stream stored audio and video contents, HTTP and FTP. Then to forwarding Multicast traffic, IP multicast group addresses were used. A multicast group address is a single IP address taken from a reserved range (224.0.0.0/4 for IPv4, FF00: /8 for IPv6) to uniquely identify a group of hosts desiring to receive certain traffic. The video source server, see figure 
(2), uses IP multicast address to setup a video conference (IPTV) session with all receivers. In multicast only a single session is setup for all receivers. The server sends only one copy of each video packet, while the router can listen to a particular group by setting the group address in the IGMP static membership information table.

The backbone, core and edge IP Multicast services must be supported by PIM-SM which is the protocol that distributes the routing information. The protocol is suitable for groups where a very low percentage of the nodes (and their routers) will subscribe to the multicast session. PIM-SM explicitly constructs a tree from each sender to the receivers in the multicast group.

At the edge where ADSL household an IP multicasting is presented that can be enabled or disabled for each IP interface. This can be configured using the attribute "IP Host Parameters->Multicast Mode".

Figures (22-24) show the performances metrics of packet loss ratio, packet jitter and end-to-end delay respectively to quantify the video streaming for VoD services which are compression by using MPEG-2 and MPEG-4.

The sender uses Unicast technology to setup a video conference session for VoD services with all receivers. In Unicast, separate sessions are setup for each receiver. Therefore, the sender sends copies of video packet, one for each receiver. While the sender uses IP Multicast technology to setup a video conference session for IPTV services with all receivers. In Multicast only a single session is setup for all receivers. Therefore, the sender sends only one copy of each video packet. Figure (25) shows Interface level example that highlighting link utilization with and without IP Multicasting.

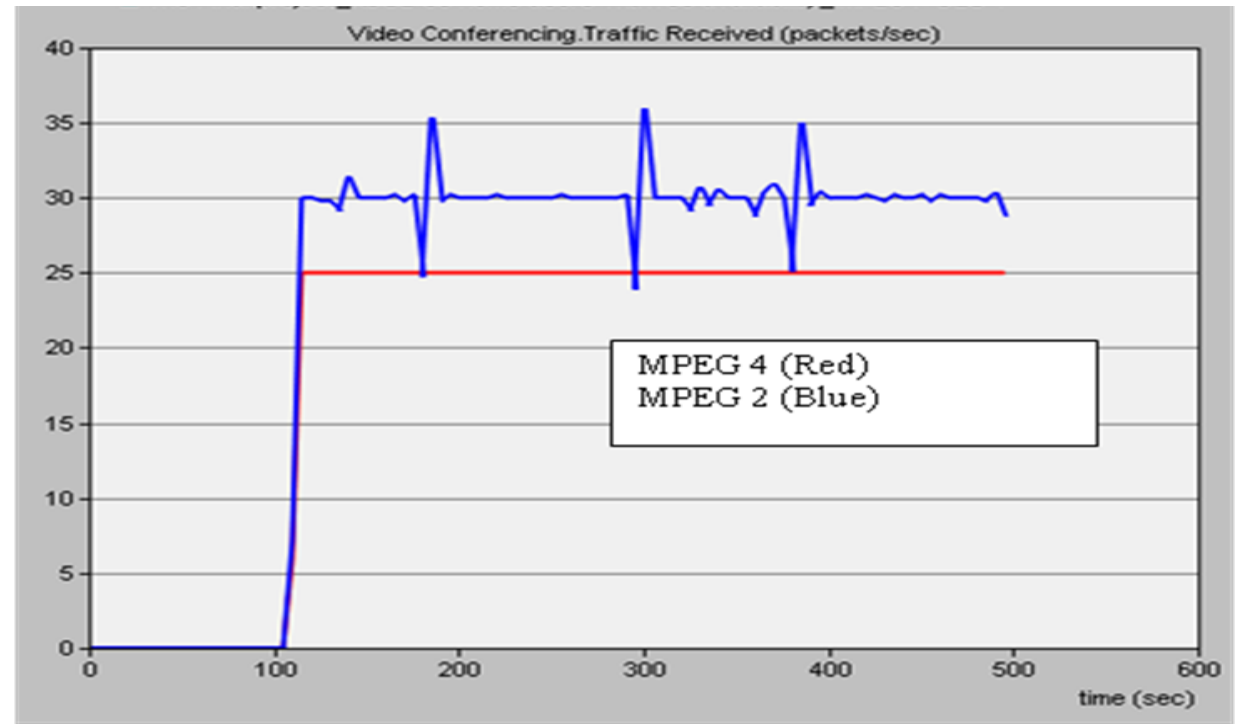

Figure 22: The MPEG-2 and MPEG-4 video PLR

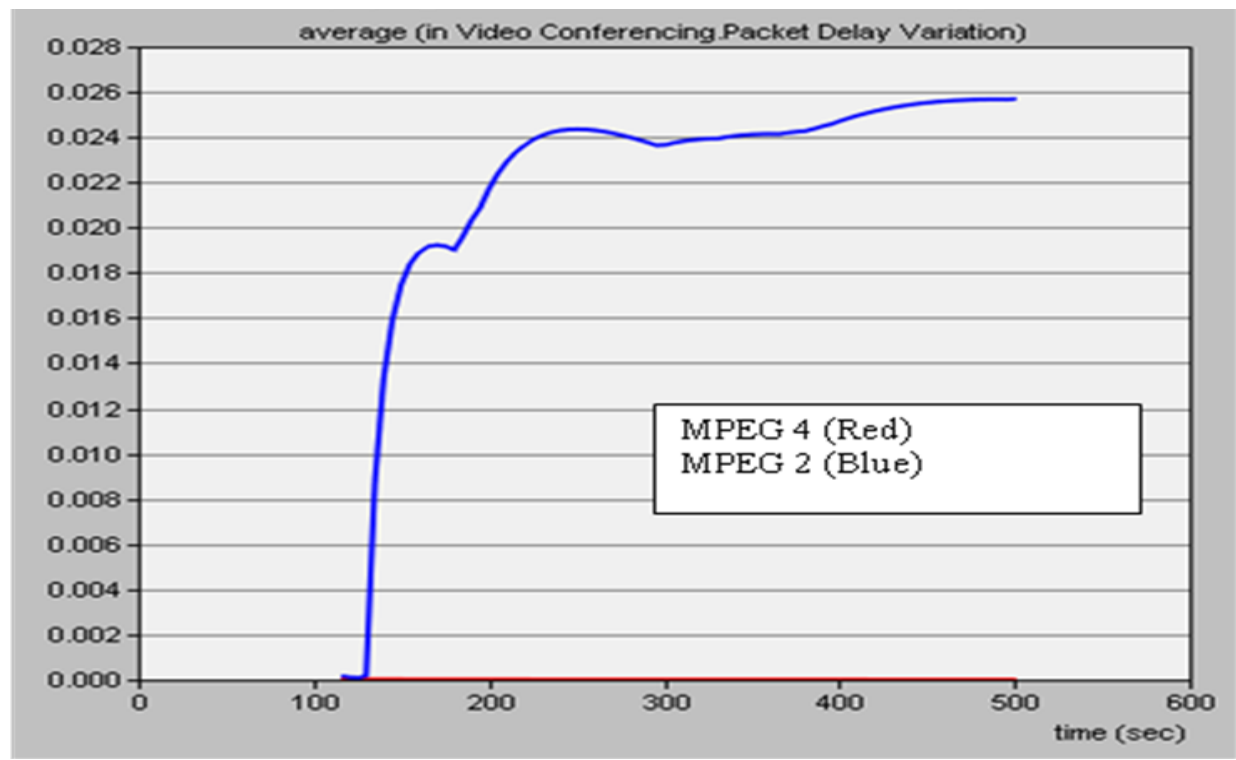

Figure 23: The MPEG-2 and MPEG-4 video packet jitter 


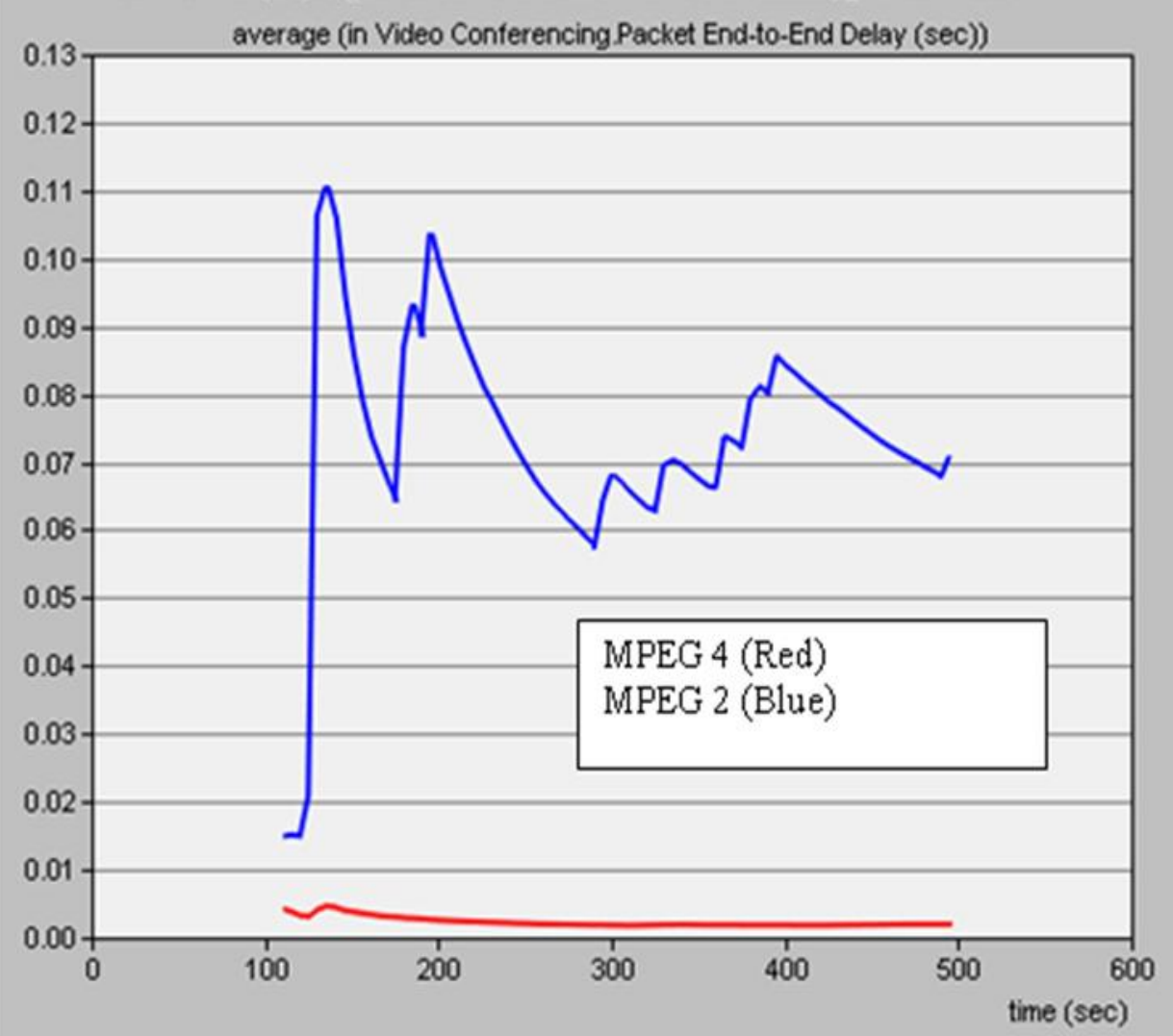

Figure 24: The MPEG-2 and MPEG-4 video end-to-end delay

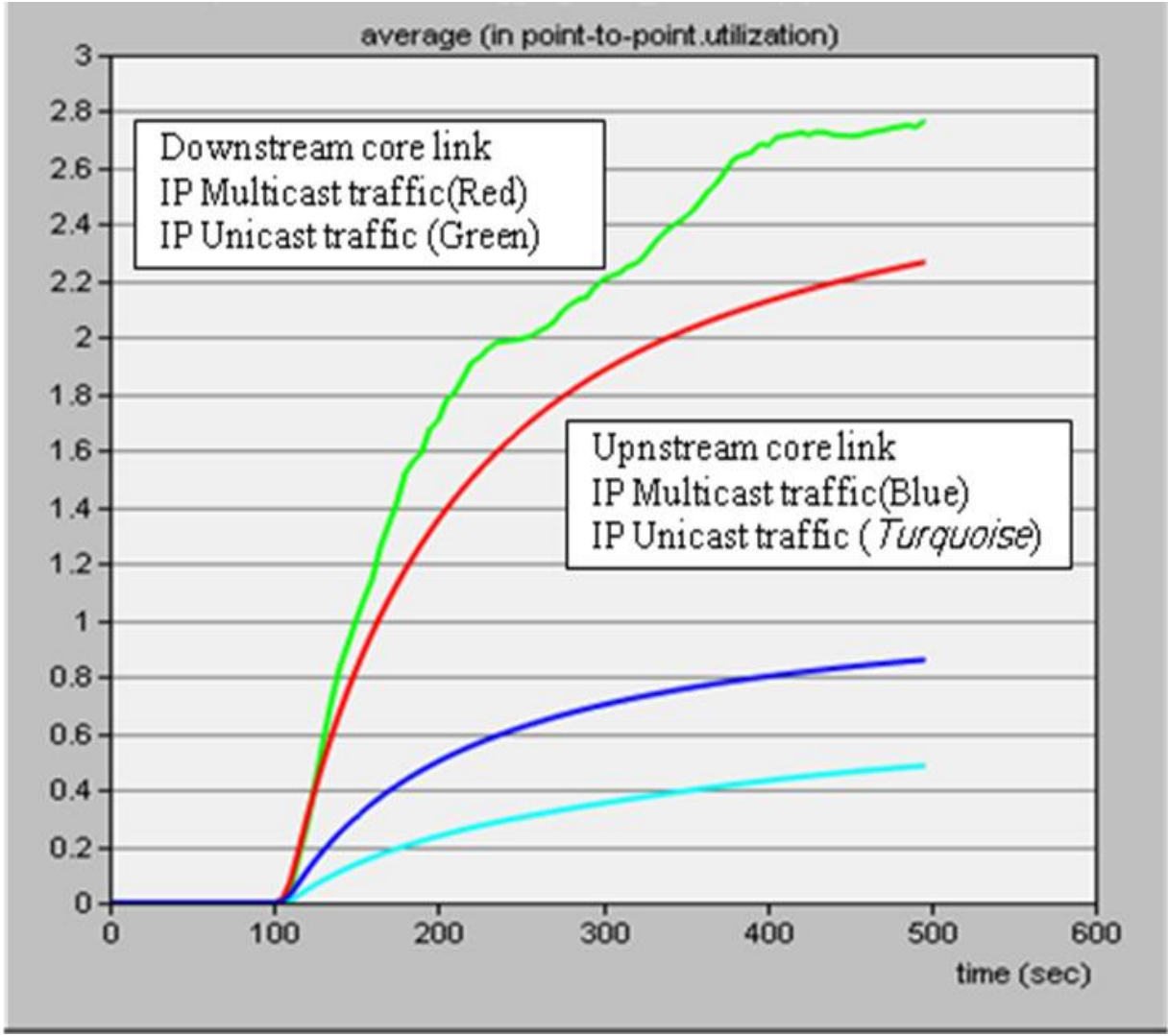

Figure 25: IP Multicast and IP Unicast Core link usage 


\section{DISCUSSION}

This work presents results of an simulations triple play transmission over hybrid network where the last mile is an ADSL supported by turbo code as FEC. The use of such a network combination is a promising service to be provided over broadband access network. The utilization of the existing telephone lines infrastructure of the user residence decreases the cost of the service. For such applications where VoD services need high bandwidth, MPEG-4 codec is the proper solution against the problems of packet loss, jitter and reordering problems. Results show, when there is available bandwidth $100 \%$, delay-variation for voice and video services are low. However, when the network availability decreases, delay-variation increases exponentially.

\section{REFERENCES}

[1] Michael G. Kallitsis," Optimal Resource Allocation for Next Generation Network Services" A PhD dissertation, North Carolina State University, 2010

[2] Borgar Torre Olsen, Dimitris Katsianis, Dimitris Varoutas, Kjell Stordahl, Jarmo Harno, Nils Kristian Elnegaard, Ilari Welling, François Loizillon, Thomas Monath, and Philippe Cadro, "Technoeconomic evaluation of the major telecommunication investment options for European players", IEEE Network, volume 20, number 4, pages 615, 2006

[3] Dr. Nasser N. Khamiss, Hothaifa Tariq Akrm, "A new proposal of digital subscriber line2 initialization process", International Journal of Advancements in computing technology, IJAT, Korea 2009.

[4] A. Neubauer, J. Freudenberger, V. Kuhn, "Coding Theory: Algorithms, Architectures and Applications", John Wiley \& Sons Ltd, England, 2007.

[5] J. Moreira, P. Farrell, "Essentials of Error-Control Coding", John Wiley \& Sons Ltd, England, 2006.

[6] Gallant, D.,"Optical network foundation for triple play services roll-out ", Optical Fiber Communication Conference, Meriton Networks, Ottawa, Ont., Canada, 2006

[7] G. Avril, F. Gauthier, F. Moulin, A. Zeddam, F. Nouvel, "Characterization of Time Variation of the Powerline Channel Frequency Response Simultaneously with Impulsive Noise", IEEE International Symposium on Power Line Communications and Its Applications, pp 330 - 335, March 2007.

[8] James F. Kurose and Keith W. Ross, "Computer Networking: A Top-Down Approach Featuring the Internet", ISBN-10: 0136079679, 9780136079675, July 10, 2010, Addison-Wesley.

[9] PATRICK SEELING, FRANK H.P. FITZEK and MARTIN REISSLEIN,"Video Traces for Network Performance Evaluation", ISBN-13 978-1-4020-5565-2 (HB), ISBN-13 978-1-4020-5566-9 (e-book), Springer 2007, 